\title{
Comparison of practical methods in clinical sites for estimating cerebral blood flow during balloon test occlusion
}

\author{
Shoichi Tani, MD, PhD, ${ }^{1}$ Hirotoshi Imamura, MD, PhD, ${ }^{1}$ Katsunori Asai, MD, PhD, ${ }^{2}$ \\ Kampei Shimizu, MD, ${ }^{3}$ Hidemitsu Adachi, MD, PhD, ${ }^{1}$ So Tokunaga, MD, ${ }^{1}$ Takayuki Funatsu, MD, ${ }^{1}$ \\ Keita Suzuki, MD, ${ }^{1}$ Hiromasa Adachi, MD, ${ }^{1}$ Shuhei Kawabata, MD, ${ }^{1}$ Yuichi Matsui, MD,1 \\ Natsuhi Sasaki, MD, ${ }^{1}$ Ryo Akiyama, MD, ${ }^{1}$ Kazufumi Horiuchi, MD, ${ }^{1}$ Chiaki Sakai, MD, PhD, ${ }^{4}$ and \\ Nobuyuki Sakai, MD, PhD'
}

\begin{abstract}
${ }^{1}$ Department of Neurosurgery, Kobe City Medical Center General Hospital, Kobe; ${ }^{2}$ Department of Neurosurgery, Osaka International Cancer Institute, Osaka; ${ }^{3}$ Department of Neurosurgery, Kyoto University Graduate School of Medicine, Kyoto; and ${ }^{4}$ Department of Neurosurgery, Hyogo College of Medicine College Hospital, Hyogo, Japan
\end{abstract}

\begin{abstract}
OBJECTIVE The authors sought to compare methods of measurement for venous phase delay (VPD) or mean stump pressure (MSTP) to rank their potential to predict ischemic tolerance during balloon test occlusion in the internal carotid artery, exploring a more correlative and convenient way to measure cerebral blood flow (CBF) that could be utilized even in the acute phase or in institutions not adequately equipped to measure CBF during the test.
\end{abstract}

METHODS X-ray angiography perfusion analysis using diagnostic digital subtraction angiography (DSA) equipment enables 1-step examination (without any room-to-room transfer of patients) to measure CBF, VPD, and MSTP completely simultaneously, which has not been accomplished by any previous perfusion studies.

RESULTS This analysis was applied to 17 patients and resulted in successful estimation of all 3 parameters in each case. The average VPD of several cortical veins had a strong correlation with relative CBF ( $\mathrm{CCBF}$ ) between bilateral hemispheres with a correlation coefficient of 0.89443 , a correlation as strong as that $(0.90357)$ of the "approximate VPD," which is interpreted based on the trend line of the scatterplot of the time to peak contrast opacification in cortical veins and their spatial positioning from the median sagittal plane. MSTP and classic visual determination of VPD have weaker correlation coefficients with $\mathrm{rCBF}(0.56119$ and 0.70048 , respectively). Overall, subjective visual determination in combination with the calculation of the trend line to estimate VPD provided a considerably strong correlation with $\mathrm{rCBF}$ $(R=0.86660)$ without any dedicated software or hardware.

CONCLUSIONS VPD has a stronger correlation with ICBF than MSTP. rCBF could be successfully predicted on common DSA equipment, even by visual determination without expensive software, if the trend line is adopted for processing to estimate VPD.

https://thejns.org/doi/abs/10.3171/2018.5.JNS18858

KEYWORDS balloon test occlusion; cerebral blood flow; ischemic tolerance; venous phase delay; mean stump pressure; correlation coefficient; vascular disorders

$\mathrm{B}$ ALLOON test occlusion (BTO) is a technique used for predicting ischemic tolerance of cerebral hemispheres and for preventing incidental strokes in the treatment of aneurysms or neoplasms that require therapeutic occlusion of a carotid artery. Presently, in this era of flow diversion, performing a BTO procedure may be declining, but it remains an important preliminary examination. Two parameters, mean stump pressure (MSTP) and venous phase delay (VPD), have been most frequently adopted at clinical sites as practical indices for ischemic hypoperfusion of cerebral blood flow (CBF) in patients undergoing BTO. ${ }^{1,713}$ Previously, MSTP and CBF were con-

ABBREVIATIONS ACA = anterior cerebral artery; $\mathrm{ACOA}=$ anterior communicating artery; $\mathrm{AUC}=$ area under the curve; $\mathrm{BTO}=$ balloon test occlusion; $\mathrm{CBF}=$ cerebral blood flow; $\mathrm{CBV}=$ cerebral blood volume; cvTTP = cortical vein TTP; DSA = digital subtraction angiography; ICA = internal carotid artery; $M C A=$ middle cerebral artery; MSTP = mean stump pressure; $\mathrm{MTT}=$ mean transit time; $\mathrm{PA}=$ posteroanterior; $\mathrm{PCOA}=$ posterior communicating artery; $\mathrm{rAUC}=\mathrm{relative} \mathrm{AUC}$; rCBF = relative $\mathrm{CBF} ; \mathrm{rMSTP}=$ relative MSTP; rMTT = relative MTT; ROI = region of interest; TTP = time to peak; VPD = venous phase delay; $\mathrm{XAP}=\mathrm{x}$-ray angiography perfusion.

SUBMITTED March 28, 2018. ACCEPTED May 16, 2018.

INCLUDE WHEN CITING Published online November 16, 2018; DOI: 10.3171/2018.5.JNS18858. 
firmed to have a correlation coefficient of 0.497 in studies measuring both variables simultaneously in patients with BTO undergoing SPECT. ${ }^{11}$ In those studies, MSTP and CBF measurements were obtained during room-to-room transfer of patients with a balloon catheter remaining inside the patients. VPD, however, cannot be detected during SPECT or other perfusion studies, and therefore the true correlation between VPD and CBF is unknown, although the correlation between VPD before the transfer and CBF after the transfer has been found to have a high correlation coefficient (0.94340). ${ }^{8}$ A novel perfusion technique, $x$-ray angiography perfusion (XAP) analysis, uses only diagnostic digital subtraction angiography (DSA) equipment to estimate CBF, VPD, and MSTP completely simultaneously. ${ }^{2}$ Through such simultaneous quantification, this study aims to compare various interpretation methods for VPD and MSTP as to their potential to predict CBF and to introduce widely available methods to any institution with no expensive software or CBF-measuring apparatus such as SPECT.

\section{Methods \\ Study Population}

Thirty-four patients underwent a total of $42 \mathrm{XAP}$ analyses with BTO for therapeutic assessment of aneurysms or other conditions at Kobe City Medical Center General Hospital from 2012 to 2017. In 25 cases MSTP was successfully measured more than 3 times with 5-minute intervals, and XAP images had sufficient resolution to allow an exact investigation of unimodal time-density curves of cortical veins, as discussed below. Four cases suffering from a carotid cavernous fistula and 4 cases with the BTO loaded in the common carotid artery were excluded because the bloodstream through the fistula or the external carotid artery might bias the pure investigation of the flow of the internal carotid artery (ICA). The remaining 17 cases were included in the study and consisted of 1 case with a skull base chordoma and 16 cases with large ICA aneurysms of various sizes, all larger than $10 \mathrm{~mm}$. There were no cases with sequelae resulting from hypoperfusion of CBF after the treatment of parent artery occlusion with or without bypass surgery, coil embolization with or without a stent, or a flow diverter. All the research was conducted according to the principles of the Declaration of Helsinki.

\section{BTO and XAP Analysis}

BTO and XAP analysis was performed as previously reported. ${ }^{2,3}$ Briefly, XAP analysis involves interpreting parameters for blood flow from the time-density curve of contrast on the DSA image of the posteroanterior (PA) view of the cranium, with contrast injected into the aorta during BTO. MSTP and mean systemic blood pressure were concurrently measured every 5 minutes during the 20-minute BTO, and the average values were used as a parameter of MSTP. A symmetric, semicircular region of interest (ROI) for XAP analysis-not including the superior sagittal sinus, transverse sinus, marginal skull and scalp, or vascular lesions such as aneurysms-was determined for detecting relative $\mathrm{CBF}(\mathrm{rCBF})$ between the right and left cerebral hemispheres (Fig. 1). Within the semicircular ROI, another symmetric triangular ROI was determined for detecting
rCBF between bilateral territories of the anterior cerebral artery (ACA) or middle cerebral artery (MCA). The boundary between the territories of the MCA and ACA was postulated to be around the line (Fig. 1, line B) that was connecting the confluence of sinuses and the middle point on the quadrant arc of the skull bone on the PA plane of the radiograph. The time-density curve within each ROI underwent XAP analysis using software for perfusion study on DSA images (2D-Perfusion, Philips Healthcare) to calculate arrival time, time to peak (TTP), mean transit time (MTT), area under the curve (AUC), etc. This 2D-Perfusion software has the additional function to set completely bisymmetric ROIs, which increases accuracy.

\section{Venous Phase Delay}

Three types of methods for estimating VPD were adopted. The first method was the classic technique using visual determination to detect contrast opacification of the first cortical veins with 3 frames per second, ${ }^{1,13}$ which was double blindly performed (and then averaged) by a neurosurgeon and two radiological technicians who had not been informed about the patients. The second method was objectively calculated on a computer, based on the timedensity curves of cortical veins. Whether the observed vessels were arterial or venous was determined according to their TTP on time-density curves compared with that of the ICA or the transverse sinus. TTP of the cortical arteries was early and close to that of the ICA, whereas TTP of the cortical veins was delayed and close to that of the sinus. On the PA image of the 2D-Perfusion software, a small, round ROI was determined within the canal of a cortical vein to provide a unimodal time-density curve, the peak of which should exhibit the TTP of contrast opacification of the cortical vein (cvTTP; Fig. 1, y). Such small ROIs were placed at more than 5 cortical veins as peripherally as possible in a hemisphere at scattered intervals. In addition, the distance of the position of the ROI from the midline (Fig. 1, x) was concurrently measured and compared to the relative width of the skull bone ( 0 to 1 for the occluded hemisphere and 0 to -1 for the nonoccluded hemisphere). The "approximate VPD by XAP" was defined as the slope of a linear function of the trend line for all scatterplots for the cvTTP, as noted on the Cartesian planes in Fig. 2, on which the x-axis represents the distance of the cortical veins from the midline of the cranium and the y-axis represents their cvTTP (the same $\mathrm{x}$ and $\mathrm{y}$ variables noted in Fig. 1). The "average VPD by XAP" was the side-toside difference of the mean of the cvTTP on 5-7 ROIs of the cortical veins in a hemisphere between the occluded and nonoccluded sides, calculated using the following equation: average VPD $=$ mean of cvTTP $_{\text {occl }}$ - mean of cvTTP $_{\text {nonoccl }}$. The third method relies on physician judgment by direct observation as follows. The "approximate VPD by eye measurement" was defined as the slope of a linear function for the trend line of all scatterplots for cvTTP, the same as that measured by XAP as mentioned above, with the exception that the cvTTP on the 5-7 ROIs was examined through visual determination on the PA image of DSA by a neurosurgeon. The neurosurgeon used a sheet with a hole as large as the diameter of a cortical vein to conceal the surroundings on the monitoring screen 

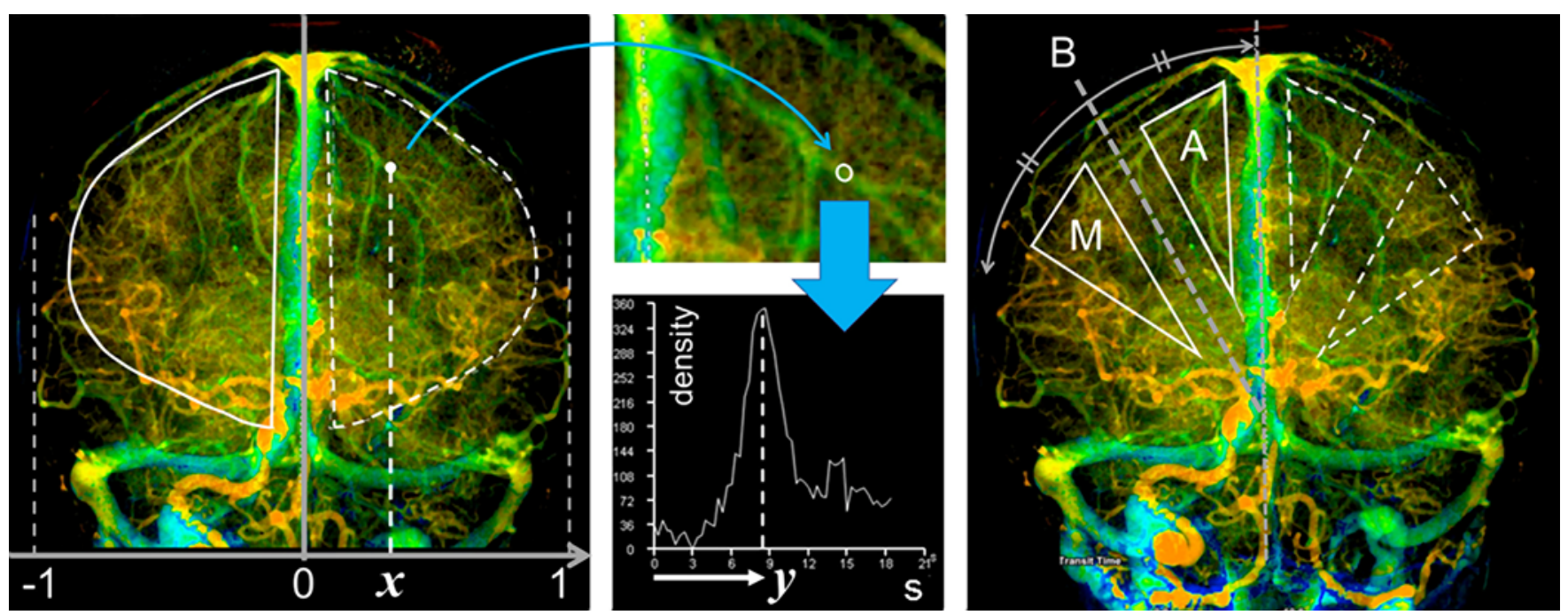

FIG. 1. XAP imaging at the BTO on the ICA. Symmetric semicircular ROls are determined for detecting rCBF between the right and left hemispheres (left panel). For detecting the TTP on the time-density curve of cortical veins $(y)$, small round ROls are located within the vessels (center panels), and the distance of the position of veins from the middle sagittal line $(x)$ is concurrently measured, while the maximum width of the skull on the occluded side is defined as 1.0 and that on the nonoccluded side as -1.0 . Symmetric triangular ROIs ( $A$ or $M$ ) for detecting rCBF between the bilateral territories of the ACA or MCA are determined above or below the hypothetical watershed borderline $(B)$, respectively (right panel). $\mathrm{s}=$ seconds. Figure is available in color online only.

to avoid any disturbance from an optical illusion by peripheral contrast opacification. The "average VPD by eye measurement" was the side-to-side difference of the mean of the cvTTP on the 5-7 ROIs of the cortical veins in a hemisphere between the occluded and nonoccluded sides, calculated with the same equation for average VPD mentioned above, with the exception that the cvTTP on the 5-7 ROIs was judged using direct observation.

\section{Statistical Analysis}

Pearson's correlation analysis was performed using Microsoft Excel to investigate the trend lines, correlation coefficients, and $\mathrm{p}$ values.

\section{Results}

\section{Spatial Distribution of VPD}

In the 17 cases with successful measurements of VPD and MSTP, the cvTTP and the position of randomly selected cortical veins were measured and plotted on the diagrams shown in Fig. 2. Whereas the time intervals of cvTTP between each cortical vein ranged widely from 0.0 to 5.8 seconds, the intervals ranged from 0.0 to 4.5 seconds if restricted within a hemisphere. The slopes of the trend lines were positive in all the detected cases and ranged from 0.31 to 2.60 seconds per hemisphere. The average cvTTP in an occluded hemisphere was invariably larger than that in a nonoccluded hemisphere in all cases.

\section{Spatial Distribution of CBF}

The relative MTT (rMTT) of semicircular ROIs covering most of hemisphere ranged from 0.590 to 0.974 . The rMTT of triangular ROIs of the ACA or MCA territory ranged from 0.603 to 1.02 or from 0.550 to 1.00 , respectively. In most cases, the rMTT of the ACA territory was higher and closer to 1.0 than that of the MCA territory (Fig. 3). The rMTT of the ACA territory in the cases with hypoplasia in the $\mathrm{A}_{1}$ portion of the ACA (designated as " $\mathrm{x}$ " in Fig. 3) appeared closer to 1.0 (ranging from 0.895 to 1.00) than that in the cases without such hypoplasia. This indicates that a more even flow was portioned between the bilateral ACA territories than between the MCA territories, and that the anterior communicating artery (ACoA) was not resistant to flow in these cases with $A_{1}$ hypoplasia.

\section{Methodological Distribution of the Correlation With CBF}

Average VPD by XAP represented a strong correlation with the rMTT of semicircular ROIs $(\mathrm{R}=0.89443$, Table 1). Approximate VPD by XAP represented a stronger correlation with the rMTT of semicircular ROIs $(\mathrm{R}=$ 0.90357). Classic VPD with visual determination represented a moderate correlation with the rMTT of semicircular ROIs ( $\mathrm{R}=0.57637,0.70434$, and 0.82073 by 3 examiners; average $\mathrm{R}=0.70048$ ). The average and approximate VPD by visual determination of cvTTP on the 5-7 ROIs in a hemisphere represented correlation coefficients of 0.78215 and 0.86660 , respectively. MSTP and relative MSTP (rMSTP) to mean systemic blood pressure (percent MSTP) represented a weak correlation with the rMTT of semicircular ROIs ( $R=0.56119$ and 0.55297 , respectively).

\section{Discussion}

$\mathrm{XAP}$ analysis is a means to estimate cerebral perfusion, which could be performed on familiar DSA equipment. This method is unique but so significant as to be cited in the DSA guidelines in the US. ${ }^{14}$ The key issue in inferring crucial physiological information from imaging of DSA is the conversion of the observed concentration time curve of injected contrast into estimates of CBF, cerebral blood vol- 

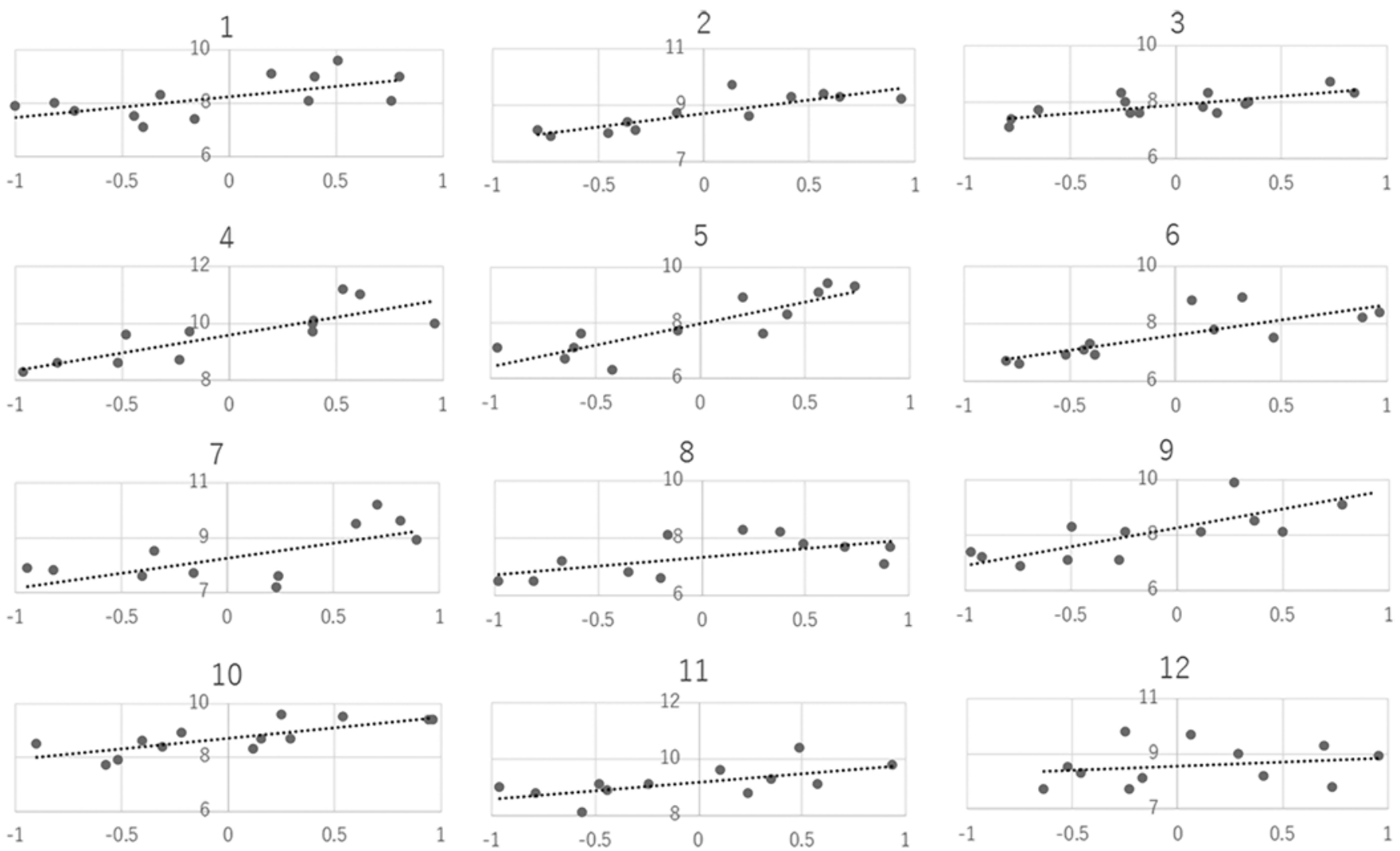

11
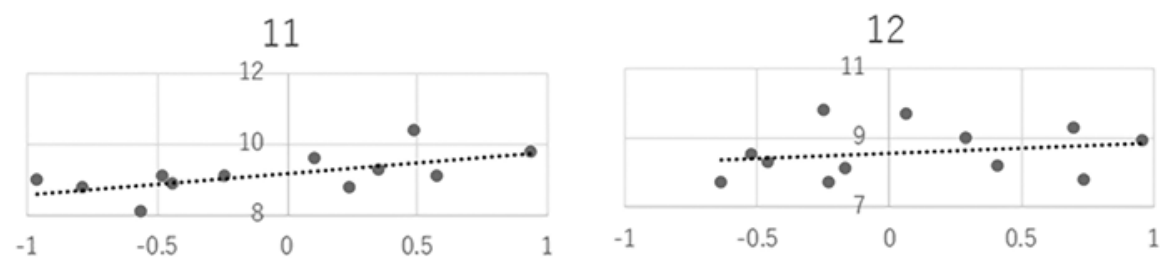

13
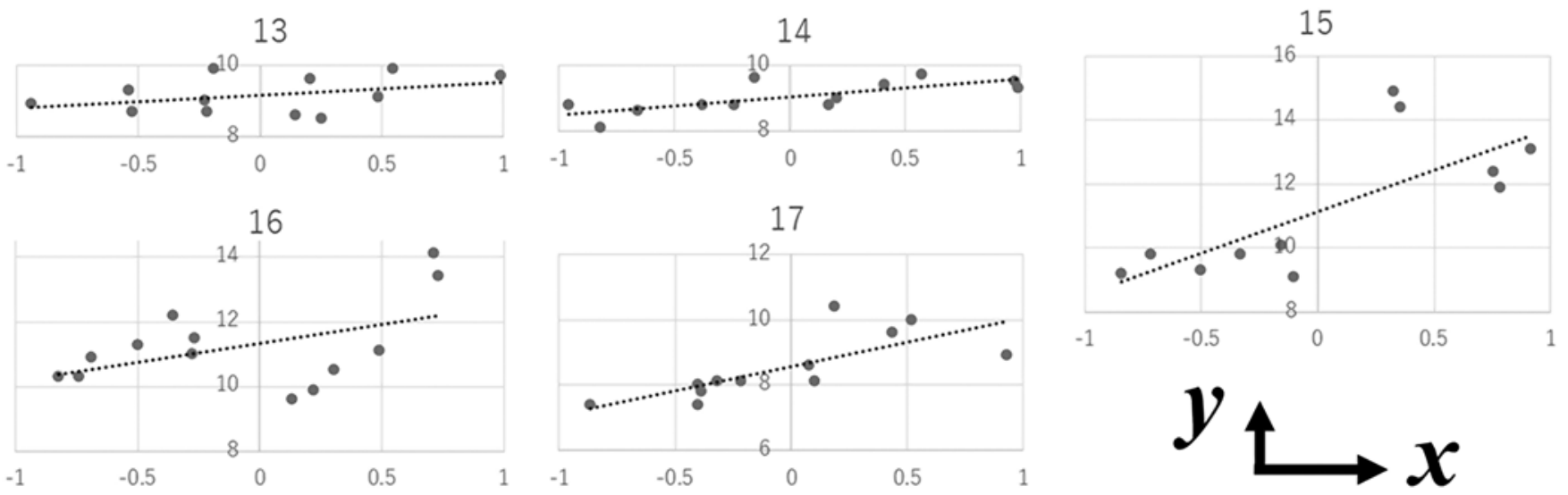

FIG. 2. Scatterplots of the position of observed cortical veins and their cVTTP in the 17 BTO cases (case number above each graph). The parameters of $x$ and $y$ are the same ones as mentioned in Fig. 1, in which the $x$-axis is the distance from the midline of the skull ( 0 to 1 for the occluded hemisphere and 0 to -1 for the nonoccluded hemisphere) and the $y$-axis is the amount of cVTTP. Each trend line is shown as a dotted line.

ume (CBV), and MTT, similar to perfusion-weighted MRI or CT perfusion studies utilizing contrast as a tracer. The investigated 2D PA view of a radiograph is not as angled as Towne's projection, but so straight $\left(0^{\circ}\right)$ that the territory of the posterior cerebral artery is located below and does not overlap with that of the ACA and MCA on the imaging plane. In addition, either the ACA or MCA could flow within such a parallel territory to the sagittal plane that each territory of the ACA or MCA could be included within a triangular space on the PA view (Fig. 1, right side). Because of the overlay of large vascular territories onto a $2 \mathrm{D}$ image, the application of XAP analysis is prone to inaccuracy depending on how the ROI is selected. Carefully selected symmetrical ROIs with respect to the sagittal plane could provide satisfactory quantitative measurement of $\mathrm{rCBF}$ on the PA view. The advantage of XAP analysis is synchronous measurement of CBF, VPD, and MSTP, which has not yet been accomplished by any other perfusion studies. Recently, rMTT measured by XAP analysis, which is the same method used in this study, has been confirmed to have a strong correlation with $\mathrm{rCBF}$ as measured by PET. ${ }^{3}$ These investigators reported that relative AUC (rAUC) measured by XAP analysis is not strongly correlative to $\mathrm{rCBV}$. If the ROI is symmetrical with respect to the sagittal 


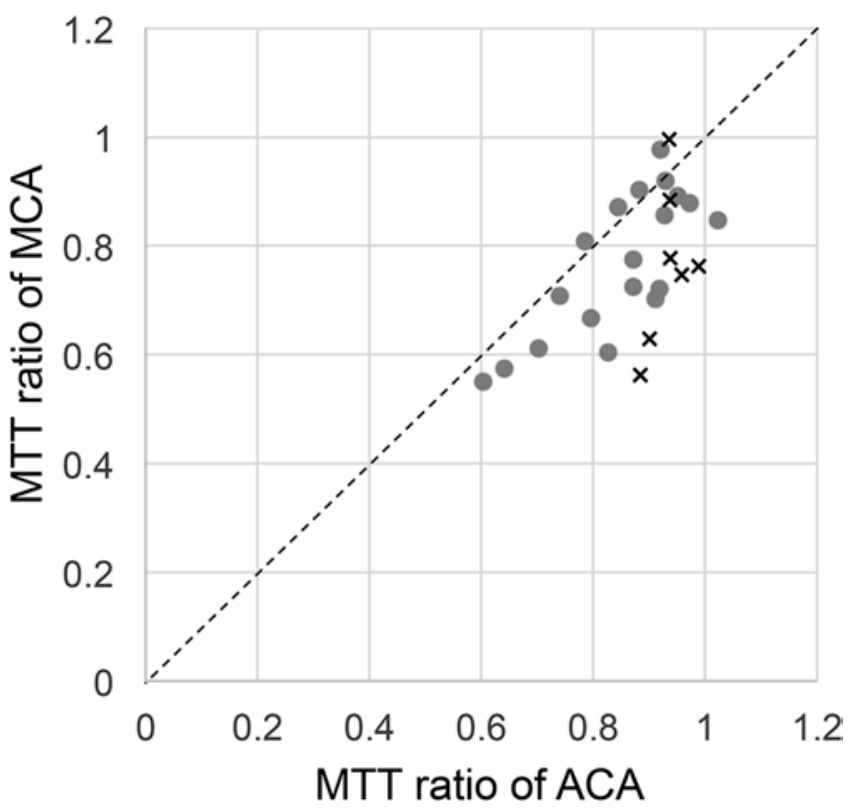

FIG. 3. Scatterplot of the relationship between the rMTT of the ACA territory and that of the MCA during BTO. Cases with an equal rate of CBF decline between the territories of the ACA and MCA in an occluded hemisphere are plotted on the dashed line (rMTT of ACA = rMTT of $M C A$ ). Cases with uneven contrast opacification around ACAs of the occluded and nonoccluded sides on the diagnostic DSA, due to aplastic or hypoplastic A, portions, are plotted as " $x$ " marks. In this diagram only, 4 cases with carotid cavernous fistula and 4 cases with BTO on the common carotid artery were added to the 17 cases shown in Fig. 2.

plane, each CBV is hypothesized to be equal, rAUC equals 1 , and rMTT equals rCBF. Therefore, rMTT of semicircular ROIs covering most of the hemisphere (as shown in Fig. 1) could represent $\mathrm{rCBF}$ between the right and left side of hemispheres perfused by ICAs, and was adopted as the index to predict ischemic tolerance during BTO in this study.

Collateral blood flow to rescue the hypoperfusion in the territory of the occluded ICA is mainly procured from two routes of the ACoA and posterior communicating artery (PCoA). ${ }^{13}$ During BTO, the collateral blood flow via the PCoA is divided into the ACA and MCA in the innate distribution ratio because this flow pours into the ICA proximal to its bifurcation toward the ACA and MCA and proceeds in the anterograde direction. Because this mode of distribution results in a similar rate of decrease in perfusion between the ACA and MCA territories, it does not cause any delayed VPD between these two areas. On the other hand, the blood flow via the ACoA could be unevenly supplied to the territories of the ACA and MCA, resulting in disparate $\mathrm{CBF}$ distribution because of the effect of hemodynamic resistance along the $\mathrm{A}_{1}$ portion of the ACA (hypoplastic or not), through which the reversed flow to the MCA should pass from the nonoccluded ICA. Including this checkpoint, there are 3 barriers for the collateral flow to pass: the $A_{1}$ portion of the nonoccluded side, the $\mathrm{ACoA}$, and the $\mathrm{A}_{1}$ portion of the occluded side, each of which may cause a gradual decrease in blood supply from the ICA on the nonoccluded side into the 4 territories (in order) of the ipsilateral MCA, the ipsilateral ACA, the contralateral ACA, and the contralateral MCA. Therefore,
TABLE 1. Ranking of various methods of interpreting VPD or MSTP for predicting CBF during BTO according to correlation coefficients with relative $\mathrm{CBF}(\mathrm{rMTT})$ and their $p$ values

\begin{tabular}{lcc}
\hline \multicolumn{1}{c}{ Method } & R Value & p Value \\
\hline XAP software on 5-7 ROls & & \\
\hline Approximate VPD & 0.90357 & $6.66 \times 10^{-7}$ \\
\hline Average in a hemisphere & 0.89443 & $1.28 \times 10^{-6}$ \\
\hline Visual determination on 5-7 ROls & & \\
\hline Approximate VPD & 0.86660 & $6.79 \times 10^{-6}$ \\
\hline Average in a hemisphere & 0.78215 & 0.000207 \\
\hline Classic visual determination* & & \\
\hline By 3 examiners & 0.70048 & 0.005697 \\
\hline Pressure transducer on the balloon catheter & & \\
\hline MSTP & 0.56119 & 0.0191 \\
\hline rMSTP & 0.55297 & 0.0213 \\
\hline
\end{tabular}

${ }^{*} R$ and $p$ values of classic visual determination are the average values among the 3 examiners.

CBF decreases in the territory of the MCA on the occluded side relative to the contralateral MCA, which should be more severe than that in the ACA. This tendency is consistent with the observation that most cases are distributed below the dashed line (rMTT of ACA = rMTT of MCA) on the scatterplot of the correlation of the rMTT of the ACA and MCA territories in Fig. 3.

This mode of divergence with gradual decreases in CBF should induce spatially correlated gradual increases in VPD along the 4 territories of the bilateral ACA and MCA, but in reality, there is too much variation in the amount of cvTTP of each region for it to be selected for the crosswise subtraction to calculate VPD. This dispersion might be because the cortical veins received some mixed flow from both the ACA and MCA territories or because the flow in the cortical veins was so slow that it took several seconds to reach the observed point. As indicated by the observation that the trend lines of cvTTP have positive slopes in all 17 cases (Fig. 2), CBF gradually declines in all cases, as it is distant from the nonoccluded side and close to the occluded side due to the 3 barriers of the ACoA and the $A_{1}$ portions. Meanwhile, when the average cvTTP in a hemisphere is used to calculate VPD between the hemispheres, the measurements of locations where cvTTP was measured within ACA or MCA territories should no longer affect the calculated value of "average VPD." Alternatively, the steepness of the slope of the trend line of cVTTP might be taken as a substitute for VPD to be designated as an "approximate VPD." This new parameter could connote the factor of spatial distribution of each cvTTP in relation to the ACA and MCA territories.

Approximate VPD either by XAP or visual determination has a strong correlation with the rMTT that represents $\mathrm{rCBF}$ between bilateral hemispheres $(\mathrm{R}=0.90357$ or 0.86660 , respectively; Fig. 4, Table 1). This authenticity is supported by the observation that the y-intercept of the trend line (1.016 or 0.9984 , respectively) is significantly close to the rMTT of 1.0, which is consistent with the theoretical prediction that when VPD is zero, bilateral CBF 

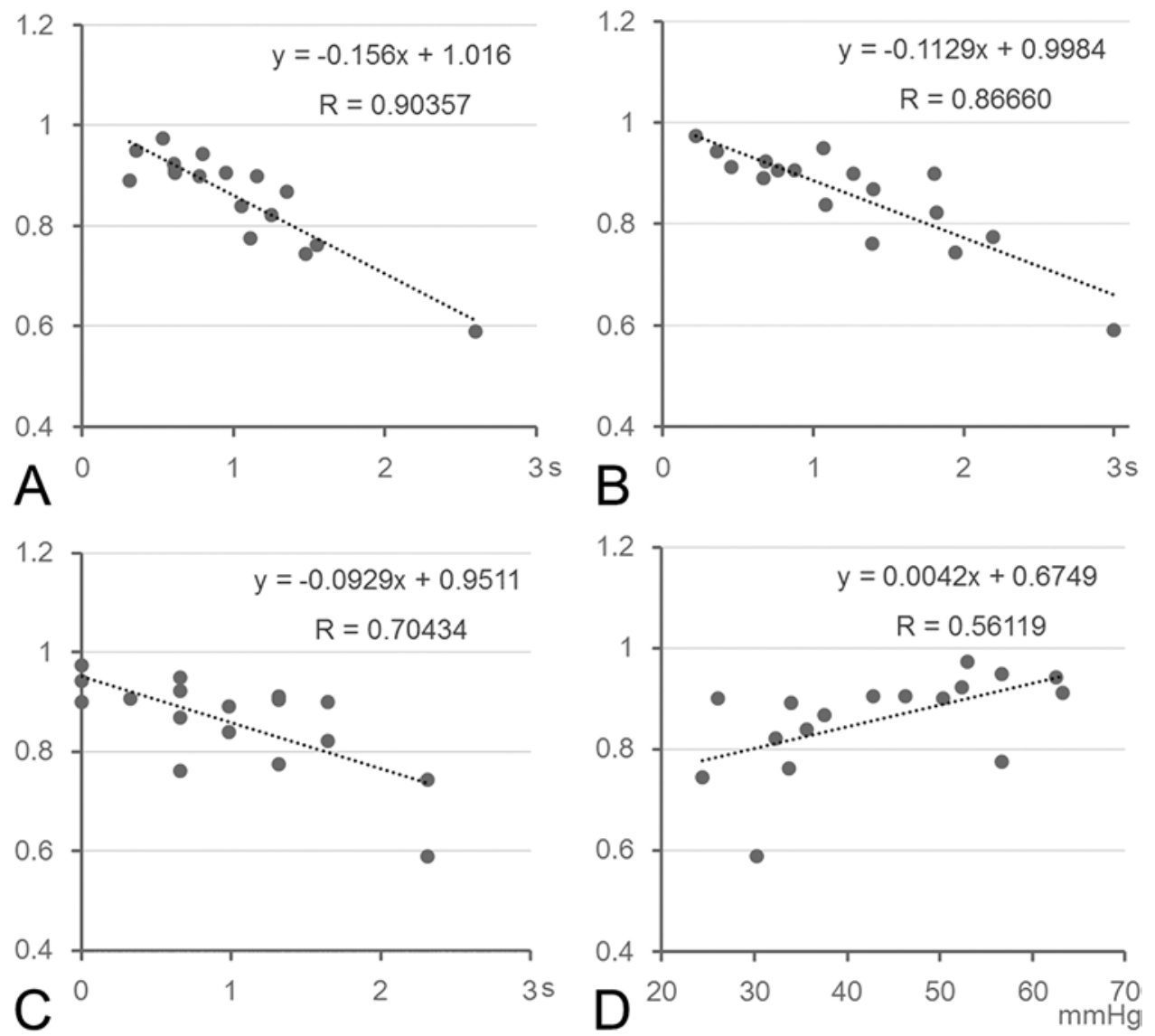

FIG. 4. Scatterplots of the correlation with $\mathrm{rCBF}$ (rMTT) regarding the 4 different parameters of approximate VPD by XAP (A), approximate VPD by visual determination (B), classic VPD (by one of the 3 examiners; C), and MSTP (D). The 17 cases introduced in this study are plotted as gray circles and their trend line is exhibited as a dotted line with the linear function and the correlation coefficient (R). The $y$-axis is the rMTT and the $x$-axis is the amount for each parameter.

measurements should be equal. The method of using the slope of the trend line might correct deviated values by visual determination, considering that the correlation coefficient of "approximate VPD by visual determination" $(0.86660)$ is appreciably higher than that of "average VPD by visual determination" (0.78215).

In contrast, the low correlation coefficient of MSTP with $\mathrm{rCBF}$ in the cases of this report $(\mathrm{R}=0.56119)$ is closely aligned with previous observations using SPECT $(\mathrm{R}=0.497),{ }^{11}$ while a correlation coefficient over 0.7 or below 0.4 is conventionally accepted as a "strong" or "weak" correlation, respectively, in statistical analysis. MSTP is therefore only weakly correlated with $\mathrm{CBF}$ despite the fact that MSTP has been generally recognized as a measure of $\mathrm{CBF}^{5,10,12}$ MSTP has been intended to reflect the blood pressure of collateral flow, but has possibly been biased for the following reasons. While the ICA is not exactly vertical to the ACA and MCA, the energy of flow from the ACA to the MCA could have been somewhat converted to the energy of pressure based on Bernoulli's principle, in a similar manner to a Pitot tube, and could thus contaminate the MSTP reading. In addition, because the flexibility of vessels should permit the back-and-forth flow even in the occluded ICA, the existence of large aneurysms would provide the ICA with sudden expansion (and contraction) of pipe flow through a stream tube, where turbulent recirculating flow causes mechanical energy loss based on the Borda-Carnot equation. Of course, the bending of the ICA and the viscosity of blood might lead to a similar kind of energy loss as well.

VPD has been widely recognized as the empirical parameter for an index of tolerance on BTO and has already been established for predicting long-term postoperative tolerance., ${ }^{4,69}$ Two disadvantages of this method are that it generally depends on the subjective direct observations of the investigators and that its exact correlation with $\mathrm{CBF}$ has not been confirmed under strictly simultaneous measurements in a perfusion study. However, as long as the potential of MSTP to predict CBF is restricted, every possible effort should be made to invent some ingenious method to increase the correlation coefficient of VPD with CBF. Two types of modifications introduced in this study, either the computational objective measurement of contrast opacification or the regression of multiple measurements to the slope of the trend lines, provide a larger correlation coefficient. The latter procedure, even by visual determination, is satisfactory for practical use in clinical sites, either in the acute phase or in poorly equipped situations, because it could be attained on the usual DSA equipment if one only calculates the slope of the trend lines using popular soft- 
ware such as Microsoft Excel and converts it into $\mathrm{rCBF}$ based on the linear function of the correlation chart.

\section{Limitations of the Study}

Assessing VPD by visual determination is flawed because it is performed visually by the investigator and thereby introduces inaccuracies. Different institutions have a slightly different time threshold of VPD predicting tolerance of the ICA according to their own outcomes of treated cases with therapeutic carotid occlusion; at our institution, patients with a VPD of more than 2 seconds, more than 1 second and less than 2 seconds, or less than 1 second were scheduled to receive high-flow bypass, superficial temporal artery to MCA bypass, or no bypass surgery, respectively. ${ }^{9}$ Carotid occlusion has been reported as tolerable in $98 \%$ of patients with a classic VPD of less than 0.5 seconds, ${ }^{6}$ with a good long-term prognosis. ${ }^{13}$ Our ways of interpreting a VPD may be sufficiently highly correlative to $\mathrm{rCBF}$ to compare them with each other for ranking, but 17 cases are too few to accurately determine the limit of VPD indicating tolerance. The $\mathrm{p}$ values of the correlation coefficient in this study are considerably low, but the data are undeniably limited by the small patient data cohort.

\section{Conclusions}

This is the first report of simultaneous measurements of VPD, MSTP, and CBF during BTO in the ICA, which were successfully attained by XAP analysis. VPD potentially has a far stronger correlation with rCBF than MSTP and could be used reliably if multiple points of cortical veins within the whole hemisphere are examined. Measuring VPD could work without any expensive hardware or software for perfusion study if clinicians interpret the results obtained by visual determination as objectively as possible, for instance, utilizing the trend line for the processing of measured amounts.

\section{Acknowledgments}

We would like to thank Shoko Masuda and Susumu Tomihari for technical assistance with the radiological examinations. We would also like to thank the staff of the Department of Research Support of Kobe City Medical Center General Hospital for preparing the article and providing guidance on statistical analysis.

\section{References}

1. Abud DG, Spelle L, Piotin M, Mounayer C, Vanzin JR, Moret J: Venous phase timing during balloon test occlusion as a criterion for permanent internal carotid artery sacrifice. AJNR Am J Neuroradiol 26:2602-2609, 2005

2. Asai K, Imamura H, Mineharu Y, Tani S, Adachi H, Narumi $\mathrm{O}$, et al: X-ray angiography perfusion analysis for the balloon occlusion test of the internal carotid artery. J Stroke Cerebrovasc Dis 24:1506-1512, 2015

3. Asai K, Nakamura H, Watabe T, Nishida T, Sakaguchi M, Hatazawa J, et al: X-ray angiography perfusion imaging with an intra-arterial injection: comparative study with ${ }^{15} \mathrm{O}$-gas/ water positron emission tomography. J Neurointerv Surg 10:780-783, 2018

4. Bechan RS, Majoie CB, Sprengers ME, Peluso JP, Sluzewski M, van Rooij WJ: Therapeutic internal carotid artery occlusion for large and giant aneurysms: a single center cohort of 146 patients. AJNR Am J Neuroradiol 37:125-129, 2016
5. Erba SM, Horton JA, Latchaw RE, Yonas H, Sekhar L, Schramm V, et al: Balloon test occlusion of the internal carotid artery with stable xenon/CT cerebral blood flow imaging. AJNR Am J Neuroradiol 9:533-538, 1988

6. Gevers S, Heijtel D, Ferns SP, van Ooij P, van Rooij WJ, van Osch MJ, et al: Cerebral perfusion long term after therapeutic occlusion of the internal carotid artery in patients who tolerated angiographic balloon test occlusion. AJNR Am J Neuroradiol 33:329-335, 2012

7. Kurata A, Miyasaka Y, Tanaka C, Ohmomo T, Yada K, Kan S: Stump pressure as a guide to the safety of permanent occlusion of the internal carotid artery. Acta Neurochir (Wien) 138:549-554, 1996

8. Sato K, Shimizu H, Inoue T, Fujimura M, Matsumoto Y, Kondo R, et al: Angiographic circulation time and cerebral blood flow during balloon test occlusion of the internal carotid artery. J Cereb Blood Flow Metab 34:136-143, 2014

9. Shimizu K, Imamura H, Mineharu Y, Adachi H, Sakai C, Tani $\mathrm{S}$, et al: Endovascular parent-artery occlusion of large or giant unruptured internal carotid artery aneurysms. A long-term single-center experience. J Clin Neurosci 37:73-78, 2017

10. Steed DL, Webster MW, DeVries EJ, Jungreis CA, Horton JA, Sehkar L, et al: Clinical observations on the effect of carotid artery occlusion on cerebral blood flow mapped by xenon computed tomography and its correlation with carotid artery back pressure. J Vasc Surg 11:38-44, 1990

11. Tomura N, Omachi K, Takahashi S, Sakuma I, Otani T, Watarai J, et al: Comparison of technetium Tc 99m hexamethylpropyleneamine oxime single-photon emission tomograph with stump pressure during the balloon occlusion test of the internal carotid artery. AJNR Am J Neuroradiol 26:1937-1942, 2005

12. Torigai T, Mase M, Ohno T, Katano H, Nisikawa Y, Sakurai $\mathrm{K}$, et al: Usefulness of dual and fully automated measurements of cerebral blood flow during balloon occlusion test of the internal carotid artery. J Stroke Cerebrovasc Dis 22:197-204, 2013

13. van Rooij WJ, Sluzewski M, Slob MJ, Rinkel GJ: Predictive value of angiographic testing for tolerance to therapeutic occlusion of the carotid artery. AJNR Am J Neuroradiol 26:175-178, 2005

14. Wojak JC, Abruzzo TA, Bello JA, Blackham KA, Hirsch JA, Jayaraman MV, et al: Quality improvement guidelines for adult diagnostic cervicocerebral angiography: update. Cooperative study between the Society of Interventional Radiology (SIR), American Society of Neuroradiology (ASNR), and Society of NeuroInterventional Surgery (SNIS). J Vasc Interv Radiol 26:1596-1608, 2015

\section{Disclosures}

The authors report no conflict of interest concerning the materials or methods used in this study or the findings specified in this paper.

\section{Author Contributions}

Conception and design: all authors. Acquisition of data: all authors. Analysis and interpretation of data: all authors. Drafting the article: Tani, Imamura. Critically revising the article: Tani. Reviewed submitted version of manuscript: all authors. Approved the final version of the manuscript on behalf of all authors: Tani. Statistical analysis: Tani. Administrative/technical/material support: Tani. Study supervision: Tani, N Sakai.

\section{Correspondence}

Shoichi Tani: Kobe City Medical Center General Hospital, Kobe, Japan.stanistanistani@herb.ocn.ne.jp. 